\title{
Analysis of vibration signal of diamond roller grinding
}

\author{
Yong Liu ${ }^{1,2}$, TingBin $\mathrm{Fu}^{1,2}$, HuaDong Zhao ${ }^{1,2}$ and ZhenWei Zhu ${ }^{1,2^{*}}$ \\ ${ }^{1}$ School of Mechanical and Power Engineering, Zhengzhou University, Zhengzhou,45000, China \\ ${ }^{2}$ Henan Institute of Intelligent Manufacturing, Zhengzhou,45000, China
}

\begin{abstract}
According to the non-linear and non-stationary characteristics of the vibration signal in the roller grinding manufacturing process, the background signals, namely the vibration signal when the grinder is idling and the vibration signal during contact grinding, are collected separately, and the signal is decomposed into multiple by the method of CEEMDAN modal decomposition. Then several components are removed by correlation analysis. Finally, the signal is denoised and analyzed by wavelet threshold denoising, which lays the foundation for the digital and intelligent grinding of diamond rollers by curve grinders. The results show that most of the grinding noise is in the low frequency, which is mainly caused by the internal friction of the machine tool; the frequency of the grinding vibration signal is mainly distributed around the two frequencies of $250 \mathrm{hz}$ and $350 \mathrm{hz}$.
\end{abstract}

\section{Introduction}

As a new generation of shaping and dressing tools, diamond rollers have the characteristics of complex contours and high machining accuracy. It is mainly used in gears, bearings, precision ball screws and other fields. The current trimming and shaping process of diamond rollers uses a curved grinder with optical magnification projection equipment to project the magnified contour on the screen. The entire grinding process relies heavily on the experience of the operator and cannot quantify the processing process.

Traditional grinding inspection mainly monitors grinding force, spindle current, temperature, vibration and other parameters, and uses artificial neural network to classify tool grinding status. For the grinding monitoring of curve grinders, domestic and foreign research focuses on how to accurately measure the wear of the grinding wheel on-line and dynamically compensate it. Wang Huai of Shanghai Jiaotong University [1] and others used the image online recognition and detection technology to realize the online detection of the processing process of the digital curve grinder. However, only relying on machine vision measurement can only obtain the size information of the processed part and the processed part, and cannot realize automation and intelligent grinding of diamond rollers. Moreover, the diamond roller itself is a tool with diamond particles enveloping its profile. The wear speed of the diamond grinding wheel for grinding the roller is much greater than that of ordinary grinding. Therefore, it is very difficult to detect and compensate the grinding wheel wear dynamically in diamond roller grinding. Compared with traditional detection methods, the acoustic emission signal is not easily interfered by environmental factors [2], has a higher signal-to-noise ratio, and can respond quickly to the transient behavior during the grinding process. Vibration signals are widely used in grinding wheel wear analysis and bearing fault diagnosis [3]. Guo Li of Hunan University [4][5] and others used wavelet analysis and GA-SVM to analyze the acoustic emission signal so that the accuracy of the judgment of the wear state of the diamond grinding wheel reached $100 \%$. Yu Teng [6] and others performed noise filtering on the extracted bridge vibration information. The experimental results show that wavelet denoising is greatly affected by the selection of wavelet basis functions. In the analysis of electrical imaging logging data, Xu Fanghui [7] and others performed wavelet threshold denoising on the high frequency IMF components decomposed by EMD, and found that the noise and interference in the output static image were significantly reduced. Kalpana Kannan [8] and others used multi-sensor fusion methods such as galvanometers and dynamometers to collect turning signals and perform time-frequency domain analysis. K. Ramasubramanian et al. used vibration and acoustic emission to analyze four different types of CVD diamond tools, and finally showed that the vibration signal is useful for analyzing the state of the tool.

Compared with Fourier transform, wavelet transform has better local feature expression ability. Limited by the principle of uncertainty, wavelet transform cannot meet the requirements of high time domain and frequency domain resolution at the same time. Based on the characteristics of nonlinear and non-stationary vibration signals in the diamond roller grinding process, this paper combines the modal decomposition method with fixed threshold filtering First, the CEEMDAN modal decomposition method is used to smooth the collected vibration signals, and then after removing several components through correlation analysis, the remaining characteristic modal functions are denoised by threshold filtering. 


\section{Vibration signal acquisition for roller grinding}

The grinder used in the test is the MK9025A CNC optical curve grinder from Shanghai No. 3 Machine Tool Factory. Use diamond grinding wheels to dress the diamond rollers. The bonding agent of the grinding wheel is ceramic, the particle size is $140 / 170$, the width is $3 \mathrm{~mm}$, and the diameter is $200 \mathrm{~mm}$. The roller used in the experiment is a forming and trimming diamond roller, which is made by electroplating, with a width of $10 \mathrm{~mm}$ and a diameter of $150 \mathrm{~mm}$. The roller is installed on the worktable of the grinder through a double-center fixture. The vibration sensor is installed on the top. During the grinding process, the temperature rise of the roller and the grinding wheel is very low, so there is no need to use the grinding fluid, which avoids the influence of the grinding fluid on the signal acquisition process. The test is carried out in the normal production grinding process. In normal grinding, the wheel speed is $4500 \mathrm{r} / \mathrm{min}$. While collecting the signal, use the camera to take a picture of the grinding area, so that the signal can be classified according to the processing state in the later stage.

As shown in Figure 1, the vibration signal generated during the grinding process passes through the ULT2001 built-in amplifier piezoelectric acceleration sensor installed on the fixture center. The frequency range of the measurement is $0.5 \sim 15000 \mathrm{~Hz}$, and the output is a voltage signal of $\pm 10 \mathrm{~V}$. The collected analog voltage signal is converted into a digital signal by the 6361 universal data acquisition card produced by NI Company, and then input to the computer for processing through LabVIEW software programming and saving as a corresponding csv file for subsequent analysis and processing in python. According

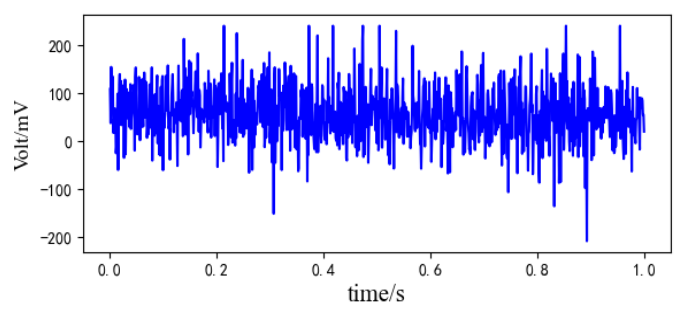

a) Idling grinding signal

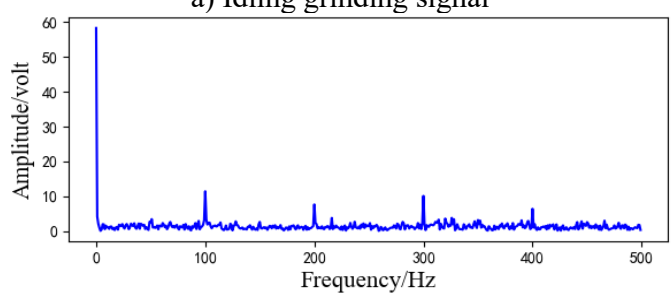

c) Grinding vibration signal to the sampling theorem, the sampling frequency of the vibration signal is set to $1 \mathrm{kHz}$.

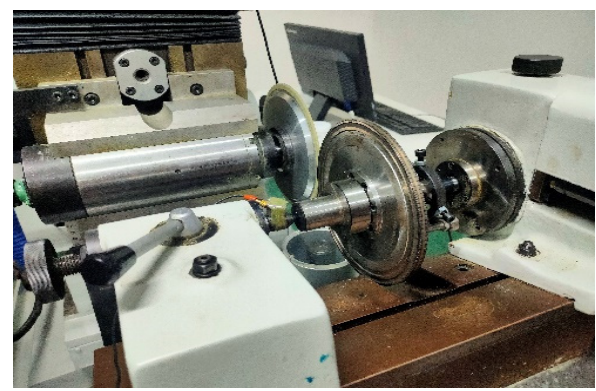

Figure 1. Installation of vibration sensor

The experimental curve grinder is placed in a factory with many processing equipment, and the vibration signals of the grinding wheel when the grinding wheel is idling and when the grinding wheel shaft is fed are respectively collected. In actual grinding, the grinding wheel is repeatedly in contact with the roller for a short period of time, and then contacts the grinding after leaving, the collected signal contains many intermittent grinding signals. Therefore, the camera is used to synchronize video recording to intercept the signal during grinding. The collected waveform and fast Fourier transform FFT spectrum analysis are shown in Figure 2. It can be seen that the frequency of the vibration signal during idling is mainly concentrated in $100 \mathrm{hz}, 200 \mathrm{hz}, 300 \mathrm{hz}$ and $400 \mathrm{hz}$. Compared with idling, the frequency of the vibration signal during grinding is $50 \mathrm{hz}, 250 \mathrm{hz}$ and $350 \mathrm{hz}$. It can be seen that the frequency of the vibration signal generated during grinding and idling is relatively evenly distributed in each frequency band, so the traditional filtering method cannot be used for noise reduction.

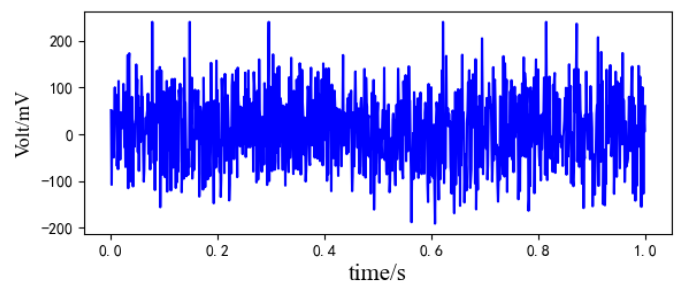

b) Idling normalized spectrogram

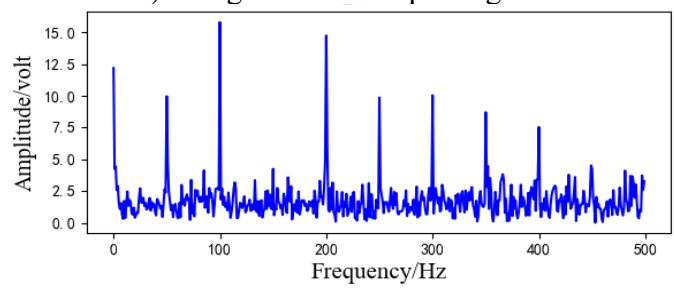

d) Grinding normalized spectrogram

Figure2. Original waveform and FFT transform spectrum diagram

\section{Wavelet threshold denoising processing based on CEEMDAN}

As an adaptive time-frequency processing method. The EMD method avoids the problem of wavelet decomposition of fixed basis functions. It is more suitable for processing nonlinear and non-stationary characteristic signals, and has the ability to characterize local transient characteristics. French engineer Flandrin came to the conclusion that under the condition of white noise, modal decomposition is equivalent to a binary filter. During modal decomposition, since the collected signal is not pure white noise, this will cause modal aliasing. In addition, modal decomposition also has end effects, mean curve 
fitting, and determination of screening stop criteria. CEEMDAN uses adaptive white noise to replace the white noise added in the traditional decomposition process. The residual noise of the decomposed components is smaller, and the interference of modal aliasing is better solved. The process of CEEMDAN decomposition is shown below:

(1) Separately add multiple adaptive white noise sequences to the original signal $S(t)$ to be decomposed, and then perform EMD decomposition on each constructed signal to obtain IMF1 and the remainder $R_{l}(t)$. The CEEMDAN IMF : $_{1}$

$$
\mathrm{IMF}_{1}=\frac{1}{i} \sum_{j=1}^{i} \mathrm{IMF}_{1}^{j}
$$

Where: $\mathrm{i}$ represents the number of times white noise is added

(2) Construct a new signal $S(t)$ to be decomposed. $S(t)=R l(t)+\lambda_{i} \sigma_{i}(t)$, where $\lambda_{\mathrm{i}}$ is the standard deviation of the noise $\sigma_{i}$ added for the $i$-th time. After decomposition, the IMF2 of CEEMDAN is:

$$
\mathrm{IMF}_{2}=\frac{1}{i} \sum_{j=1}^{i} \mathrm{IMF}_{2}^{j}
$$

(3) Repeat (1) and (2) until the termination conditions are met and CEEMDAN decomposition is completed

As shown in Figure 3, CEEMDAN decomposition of the signal. The idling signal has 7 components, and the grinding vibration signal has 8 components.

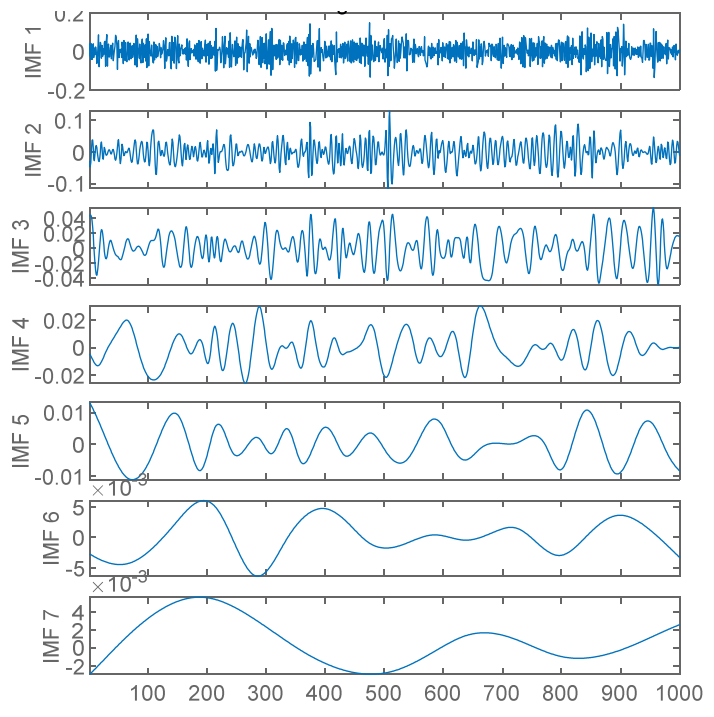

Figure3. Modal decomposition of idling vibration signal

The result of modal decomposition is a set of IMFs with the center frequency from high to low. The idea of modal decomposition and denoising is to reconstruct the signal after removing several high-frequency IMF components, which can be regarded as high-frequency filtering; in the same way, removing the low-frequency components and reconstructing the signal can be regarded as low-frequency Filtering. However, when the noise signal exists in both high-frequency and low-frequency IMF components, removing too many IMF components will lose a lot of useful information. At this time, the two components with the lowest correlation coefficient can be removed through correlation analysis, and then the wavelet threshold denoising of the remaining IMF components can solve this problem well. Here, the SNR of the signal before and after denoising is used to evaluate the filtering effect. The calculation formula of SNR is:

$$
S N R=10 \log \left[\frac{\sum_{i=1}^{N} x^{2}(j)}{\sum_{j=1}^{N}[x(j)-\bar{x}(j)]^{2}}\right]
$$

After CEEMDAN modal decomposition, several IMF components will be obtained. It is often considered that the first component represents the highest frequency component of the signal, and the remaining components represent the average trend of the signal. The degree of correlation between these components and the original signal represents the proportion of this component to the effective components in the original signal [15]. Therefore their correlation coefficients can be calculated separately, and the calculation formula is:

$$
\rho_{X, Y}=\frac{E(X Y)-E(X) E(Y)}{\sqrt{E\left(X^{2}\right)-(E(X))^{2}} \sqrt{E\left(Y^{2}\right)-(E(Y))^{2}}}
$$

Figure 4 shows the correlation coefficient of the components obtained by the decomposition of the idling and grinding signals. It can be seen that the correlation coefficient of IMF6 component is the lowest during idling and grinding. In this way, the threshold denoising can be performed on other modal components with the lowest correlation component removed.

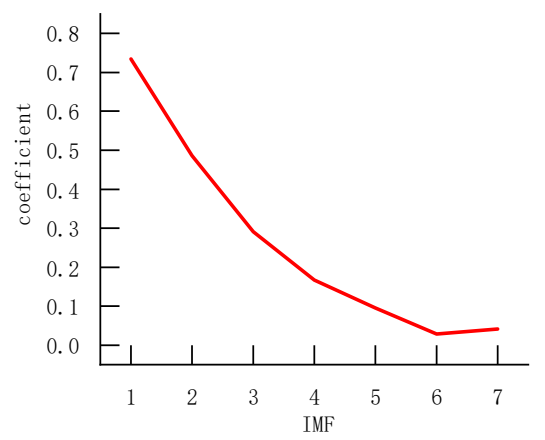

a) Correlation coefficient of idling signal

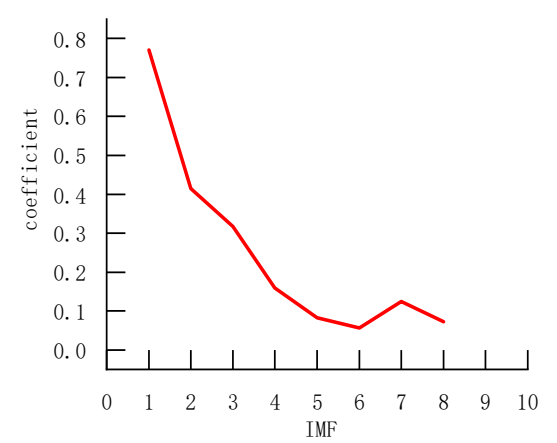

b) Correlation coefficient of grinding signal

Figure4. Modal component correlation coefficient

After modal decomposition, fixed threshold denoising or wavelet denoising is often used to analyze the signal. The fixed threshold denoising is to select a value to cut off each component separately. The threshold has a great influence on the result of denoising. Calculated as follows:

$$
\lambda=\sigma \sqrt{2 \ln (N)}
$$


In the formula: $\sigma$ is the standard deviation of the noise. Generally, the first IMF component decomposed is used as the estimated noise. Here we take the collected background signal, that is, the idling signal as the estimated noise; $\mathrm{N}$ is the length of the sample.

Wavelet threshold denoising is to remove the wavelet coefficients related to noise, and finally reconstruct with inverse transform. Two wavelets, $\mathrm{db} 4$ and sym4 are

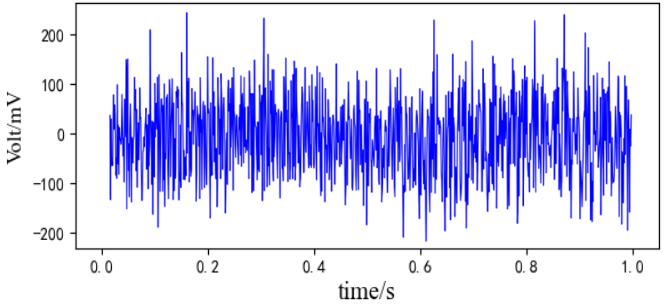

a) Idling noise reduction signal selected here, the threshold function is a new wavelet threshold function, and the threshold is a fixed threshold. The denoising effect is shown in Table 1, where SNR1 is the signal-to-noise ratio of the idling signal, and SNR2 is the signal-to-noise ratio of the grinding signal. It can be seen that the sym 4 wavelet has the best effect. The signal after denoising is shown in Figure 5, and the SNR after denoising is shown in Table 1.

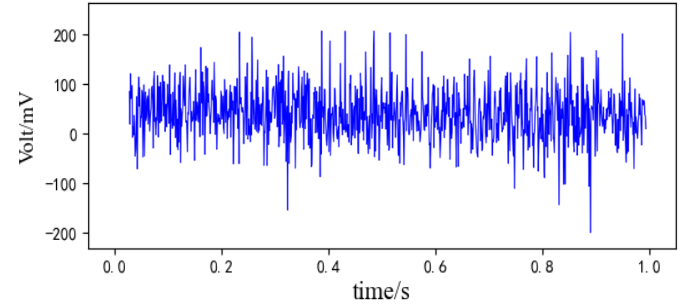

b) Correlation coefficient of grinding signal

Figure5. Noise reduction signal

TABLE.1 The noise reduction effect of different methods is compared

\begin{tabular}{ccc}
\hline Species & SNR1 & SNR2 \\
\hline Fixed threshold & 6.52 & 4.34 \\
db4 & 31.49 & 34.78 \\
sym4 & 43.44 & 42.69 \\
\hline
\end{tabular}

\section{Conclusions}

In order to monitor the status of roller grinding, the vibration signal is analyzed with modal decomposition combined with wavelet threshold denoising. Compared with the idling vibration signal, the grinding vibration signal has more frequencies of $50 \mathrm{hz}, 250 \mathrm{hz}$ and $350 \mathrm{hz}$. From the correlation analysis, it can be seen that the lowfrequency signal is a noise signal, which is caused by the internal friction of the machine tool. This part of the noise filtering can be achieved by directly removing the corresponding modal components. The filtering of noise scattered in other frequency bands needs to be realized by threshold denoising. After comparison, it is found that the sym 4 wavelet base has the best effect, while the simple use of a fixed threshold denoising method has the worst effect.

For the denoised signal, the next step can be to calculate the effective value, energy coefficient and variance value combined with the grinding state of the signal to establish a grinding data set, and use the support vector machine to predict and classify the grinding state. The data set can also be established by calculating the relevant parameters of each modal component after wavelet threshold denoising, and the roller grinding model can be established by using CNN or RNN.

\section{Acknowledgments}

This paper was completed under the guidance of Professor Huadong Zhao and Dr. Zhenwei Zhu, thank them for their selfless help. Thanks for the platform provided by Henan Intelligent Manufacturing Research Institute. Thanks for the support of the key technology development and industrialization project of complex diamond products with high precision (18XTZX12006).

\section{References}

1. Wang Huai. Research on key Technologies of Digital precision curve Grinding based on online image recognition and detection [D].Shanghai Jiao Tong University, 2008.

2. Fan Linlin. Application of Acoustic emission technology in nondestructive testing [D]. Harbin University of Science and Technology,2010.

3. Tian Rui. Study on detrending analysis and Fault Feature Extraction method of bearing vibration Signal [J]. Mechanical Design and Manufacturing, 2018(12):100-104.

4. Guo Li, Li Bo, Guo Juntao.Acoustic Emission Monitoring of Diamond Wheel Wear based on wavelet analysis and GA-SVM [J].Mechanical and electrical engineering,2019,36(12):1255-1260.

5. Guo Li, Huo Ke, Guo Juntao. Acoustic emission monitoring of wear state of diamond grinding wheel based on EMD [J]. Journal of hunan university (natural science edition),2019,46(02):58-66.

6. Yu Teng, HU Wu-sheng, WU Jie, LI Hai-feng, QIAO Yan. Extracting vibration information of Runyang Bridge based on wavelet threshold denoising and EMD decomposition [J].Vibration and impact, 2019, 38(12):264-270.

7. $\mathrm{Xu}$ Fang-hui, WANG Zhu-wen, LIU Jing-hua, OU Wei-ming.Joint EMD and wavelet threshold denoising in electrical imaging logging data [J]. Journal of China university of petroleum (natural science edition),2020,44(03):56-65.

8. Kalpana Kannan, N.Arunachalam,Aakash Chawla, Sundararajan Natarajan. Multi-Sensor Data Analytics for Grinding Wheel Redress Life Estimation- An Approach towards Industry $4.0[\mathrm{~J}]$. Procedia Manufacturing, 2018,26. 